\title{
Precision, volume and eigenspectra for GPS ambiguity estimation based on the time-averaged satellite geometry
}

\author{
P. J. G. Teunissen \\ Delft Geodetic Computing Centre (LGR), Faculty of Geodesy, Delft University of Technology, Thijsseweg 11, 2629 JA Delft, \\ The Netherlands
}

Received: 3 April 1996; Accepted: 6 January 1997

\begin{abstract}
In this contribution we consider the timeaveraged GPS single-baseline model and study in a qualitative sense its relation with the geometry-free model and the geometry-based model. The least-squares estimators of the model are derived and their properties discussed. Special attention is given to the ambiguity search space, since it plays such a crucial role in the problem of integer ambiguity estimation and validation. Easy-to-evaluate, closed-form expressions are presented for the volumes of the ambiguity search spaces that belong to the geometry-free model, the single-epoch geometry-based model and the time-averaged model. By means of an eigenvalue analysis, the geometry of the ambiguity search spaces is revealed and its impact on the search for the integer least-squares ambiguities discussed.
\end{abstract}

Key words. GPS, ambiguity, volume

\section{Introduction}

The various approaches in use for integer ambiguity estimation can be distinguished as to whether they make use of the available relative receiver-satellite geometry or not. Approaches that make use of the receiver-satellite geometry are usually the common mode of operation in surveying applications. They allow for instantaneous relative positioning, depending on whether both code and phase data or only phase data are used. Examples of such approaches can be found in Blewitt (1989), Frei and Beutler (1990), Hatch (1991), Teunissen (1993) and Tiberius and de Jonge (1995). Integer ambiguity estimation is also possible however when one opts to dispense with the receiver-satellite geometry. In fact this is the simplest approach to integer ambiguity estimation. The code data are almost directly used to determine the unknown integer ambiguities of the observed phase data. Examples of such approaches can be found in Hatch (1982), Euler and Goad (1990), Dedes and Goad (1994), Euler and Hatch (1994) and Teunissen (1996).

We will refer to the model used by the first class of approaches as the geometry-based model and to the model used by the second class of approaches as the geometry-free model. In this contribution, we also consider the time-averaged model; it is a time-averaged version of the geometry-based model, but has many properties in common with the geometry-free model. The rationale for considering this model is the slowly changing GPS relative receiver-satellite geometry. It is therefore of interest to study the properties of the estimators that are based on the time-averaged model and in particular of the corresponding estimates of the integer ambiguities. Furthermore, since the single-epoch geometry-based model is a special case of the time-averaged model, the results obtained for the latter model are directly applicable to the former. The results are therefore also of relevance for instantaneous relative positioning.

The models considered are of the single-baseline type, separated by a short distance only. The term 'short' refers to the assumption that the double-differenced GPS observables are sufficiently insensitive to orbital uncertainties in the fixed orbits and to residual ionospheric and tropospheric delays. We also assume that time correlation is absent and that no correlation exists between the phase and code observables. So as to obtain a deeper understanding of the intricacies involved in ambiguity resolution, this contribution presents a qualitative, rather than a quantitative analysis.

In Sect. 2 the time-averaged model is introduced and its least-squares estimators are derived. The differences and similarities between these estimators and those of the geometry-free model and geometry-based model are discussed. This is done both for the single-frequency case as well as for the dual-frequency case.

Several characteristics of the ambiguity search space, which play a central role in the problem of integer am- 
biguity estimation, are discussed in Sect. 3. In Sect. 3.1 we present easy-to-evaluate, closed-form expressions for the volume of the ambiguity search space. This is done for both the time-averaged model as well as for the geometry-free model. The volume can be used appropriately to scale the size of the ambiguity search space and it provides an estimate for the number of grid points inside the search space. The volume formulae presented clearly show which role is played by the three types of data redundancy: the number of observation epochs used, the number of satellites tracked and the number of frequencies observed.

In Sect. 3.2 we discuss the precision of the widelane ambiguities and show that it is not guaranteed that the widelane ambiguities are of a better precision than the $L_{1}$ and $L_{2}$ ambiguities. It is shown under which conditions which functions of the widelane ambiguities are of a better precision than the same functions of the $L_{1}$ ambiguities.

In Sect. 3.3 we present the principal axes geometry of the ambiguity search spaces. This analysis allows us to show how the efficiency of the search for the integer least-squares ambiguities can be improved by transforming the original double-differenced ambiguities to new and less correlated ambiguities.

\section{The time-averaged single-baseline model}

In this section we introduce the time-averaged singlebaseline model. We consider short baselines and therefore assume that the ionospheric delays are absent or sufficiently small to be neglected. We also assume that the same $m$ satellites are tracked during the observation time-span. This is reasonable for short observation timespans, as used in fast ambiguity resolution applications. As a result, it allows the derivation of closed-form formulae, which give an in-depth understanding of the various factors that contribute to ambiguity resolution.

The time-averaged model is positioned in between the geometry-free model and the geometry-based model. The least-squares estimators of the baseline and the ambiguities of the time-averaged model are derived and compared with their counterparts of the geometry-free model. The least-squares estimators of the geometry-free model will be indicated with a tilde " ." and the leastsquares estimators of the time-averaged model will be indicated with a hat " .". We reserve the check-sign " ." for the fixed baseline. We consider both the single- and the dual-frequency case.

\subsection{The single-frequency case}

First we will consider the geometry-free model, then the geometry-based model and finally the time-averaged model.

2.1.1 Geometry-free model The single-baseline model that dispenses with the receiver-satellite geometry reads
$D^{T} \phi_{1}(i)=D^{T} r(i)+\lambda_{1} a_{1}$

$D^{T} p_{1}(i)=D^{T} r(i)$

for $i=1, \ldots, k$. The epoch number is denoted by $i$ and the total number of epochs equals $k$. The singlefrequency, single-differenced (SD) phase and code observables of epoch $i$ are collected in, respectively, the $m$ vectors $\phi_{1}(i)$ and $p_{1}(i)$. The $(m-1) \times m$ matrix $D^{T}$ transforms the SD observables into double-differenced (DD) observables. Note that the phase observables have been expressed in units of range, rather than in units of cycles. The unknown integer DD ambiguities on $L_{1}$ are collected in the $(m-1)$-vector $a_{1}$, where $\lambda_{1}$ is the known wavelength of $L_{1}$. The $m$-vector $r(i)$, contains the unknown SD forms of the $m$ receiver-satellite ranges at epoch $i$. The DD vector $D^{T} r(i)$ is of course treated as the parameter vector and not $r(i)$, since we would otherwise have a rank deficiency in the design matrix. The matrix $D^{T}$ is shown explicitly though, since it will play an important role in our further analysis.

The variance matrices of $D^{T} \phi_{1}(i)$ and $D^{T} p_{1}(i)$ are given as $\sigma_{\phi}^{2} D^{T} D$ and $\sigma_{p}^{2} D^{T} D$, where $\sigma_{\phi}^{2}$ is the variance of the SD phase observable and $\sigma_{p}^{2}$ is the variance of the $\mathrm{SD}$ code observable. Time correlation is assumed absent and no correlation is assumed to be present between the phase and code observables. Also the ionospheric delays are assumed absent or sufficiently small to be neglected. The impact of the ionospheric delays, including their weighted form, has been studied in Teunissen (1997).

The following features of the geometry-free model are noteworthy. First, the receivers need not be stationary per se. Second, tropospheric delays will get lumped with the range parameters. Hence, the leastsquares ambiguity estimators are not affected by these delays when present. Third, code data are needed per se to be able to solve for the ambiguities. Fourth, there is no redundancy when single epochs are considered. Hence, the redundancy enters only when more than one epoch is considered. For $k$ epochs, the redundancy equals $(m-1)(k-1)$. Since there is no redundancy per epoch, the least-squares estimate of the ambiguity vector follows as the time-average of the ambiguity estimates computed per epoch. Since the ambiguity estimate of a single epoch is given as $a_{1}(i)=D^{T}\left[\phi_{1}(i)-p_{1}(i)\right] / \lambda_{1}$, the least-squares estimate follows after averaging over time as

$\tilde{a}_{1}=\frac{1}{\lambda_{1}} D^{T}\left[\bar{\phi}_{1}-\bar{p}_{1}\right]$

with the time averages $\bar{\phi}_{1}$ and $\bar{p}_{1}$. The ambiguity variance matrix follows from applying the error propagation law. It reads

$Q_{\tilde{a}_{1}}=\frac{\sigma_{\phi}^{2}}{\lambda_{1}^{2} k}\left(1+\frac{1}{\epsilon}\right) D^{T} D$

with the phase-code variance ratio $\epsilon=\sigma_{\phi}^{2} / \sigma_{p}^{2}$.

Since the precision of the code observables is much poorer than that of the phase observables, $\epsilon$ will be very small in practice $\left(e . g . \epsilon \simeq 10^{-4}\right)$. Hence, the precision of 
the ambiguities will be rather poor, unless $k$ is large enough. Successful validation of the ambiguities can therefore be expected only if a sufficient number of observation epochs are taken into account.

2.1.2 Geometry-based model Let $A(i)$ be the $m \times 3$ SD design matrix that captures the relative receiver-satellite geometry at epoch $i$ and let $b$ be the 3-vector that contains the unknown increments of the three-dimensional baseline. Then, after linearization, $r(i)=A(i) b$ (remark: from now on the range vector and the phase and code observables in the linear model Eq. 1, should be read, respectively, as an unknown increment or as 'observed minus computed'). Substitution into Eq. (1) gives the geometry-based model as

$$
\begin{aligned}
& D^{T} \phi_{1}(i)=D^{T} A(i) b+\lambda_{1} a_{1} \\
& D^{T} p_{1}(i)=D^{T} A(i) b
\end{aligned}
$$

for $i=1, \ldots, k$. Since $b$ is time invariant, whereas $r(i)$ is not, the number of unknown parameters has decreased from $(m-1)(k+1)$ to $m+2$. The redundancy is therefore now equal to $(m-1)(2 k-1)-3$. Singleepoch solutions are therefore possible when $m \geq 4$ (configuration defects in the receiver-satellite geometry are assumed to be absent). If code data are absent, the redundancy equals $(m-1)(k-1)-3$. In this case a single-epoch solution is not possible, but a double-epoch solution is, provided that $m \geq 4$. Hence, in contrast with the geometry-free model, code data are not needed per se.

2.1.3 Time-averaged model This model follows from taking the time-average of the vectorial observation equations of Eq. (4). It reads

$$
\begin{aligned}
D^{T} \bar{\phi}_{1} & =D^{T} \bar{A} b+\lambda_{1} a_{1} \\
D^{T} \bar{p}_{1} & =D^{T} \bar{A} b
\end{aligned}
$$

where $\bar{A}$ is the time-average of $A(i)$. The variance matrices of $\bar{\phi}_{1}$ and $\bar{p}_{1}$ are given as $\sigma_{\phi}^{2} D^{T} D / k$ and $\sigma_{p}^{2} D^{T} D / k$.

Our motivation for studying the time-averaged model is twofold. First, since the GPS receiver-satellite geometry is known to change slowly with time, the results that hold true for the time-averaged model should not differ too much from the results that hold true for the geometry-based model, at least when short observation timespans are considered, as is the case with fast ambiguity resolution applications. In fact, the time-averaged model is identical to the geometry-based model when $k=1$. This implies that our derivations automatically provide, free of charge, the results that hold true for the singleepoch geometry-based model. These results are therefore in particular of relevance for instantaneous relative positioning. Second, the time-averaged model also has many properties in common with the geometry-free model. In fact, the time-averaged model is positioned in between the geometry-free and the geometry-based model. Note that the output data of the geometry-free model, the time-averaged observables, are the input data for the given model.

The unknown parameters of the time-averaged model are identical to the unknown parameters of the geometry-based model. However, when $k>1$, the number of observables has been reduced. Hence, also the redundancy has been reduced. It equals $(m-4)$ and is solely due to the presence of the code data. Just as with the geometry-free model, code data are needed per se to be able to solve for the model. Without code data, the design matrix of the time-averaged model has a rank defect of 3 .

Since code data are needed per se to solve for the time-averaged model, the least-squares solution for the floated baseline will be independent of the phase data. Hence, the floated baseline will be a pure code-based solution. Its least-squares solution, together with its variance matrix, are given as

$$
\begin{aligned}
& \hat{b}=\left(\bar{A}^{T} P \bar{A}\right)^{-1} \bar{A}^{T} P \bar{p}_{1} \\
& Q_{\hat{b}}=\frac{\sigma_{p}^{2}}{k}\left(\bar{A}^{T} P \bar{A}\right)^{-1}
\end{aligned}
$$

The matrix $P$ is an orthogonal projector that projects onto the range space $R(D)$ and along the null space $N\left(D^{T}\right)=R\left(e_{m}\right)$, where $e_{m}$ is the $m$-vector having 1 in all its entries. The presence of the projector $P$ is due to the double-differencing process involved. The projector can be expressed in the following two ways

$P=D\left(D^{T} D\right)^{-1} D^{T}=I_{m}-\frac{1}{m} e_{m} e_{m}^{T}$

In the floated baseline solution we see two types of average at work; by definition a time-average in the code data $\left(\bar{p}_{1}\right)$ and in the receiver-satellite geometry $(\bar{A})$. But due to the presence of $P$, there is also a satellite averaging involved. Since $I_{m}-P$ projects orthogonally onto the vector having 1 in all its entries, residuals are formed of the single-differenced variables with respect to their average over all $m$ satellite channels.

The fixed-baseline solution can be obtained once the ambiguities are assumed to be known. In contrast to the floated baseline, the fixed baseline will depend on both the code and phase data. Its least-squares solution and variance matrix are given as

$$
\begin{aligned}
& \check{b}=\left(\bar{A}^{T} P \bar{A}\right)^{-1} \bar{A}^{T} P \bar{q}_{1} \\
& Q_{\breve{b}}=\frac{\sigma_{p}^{2}}{k} \frac{\epsilon}{1+\epsilon}\left(\bar{A}^{T} P \bar{A}\right)^{-1}
\end{aligned}
$$

with the SD vector

$\bar{q}_{1}=\left[\sigma_{p_{1}}^{2}\left(\bar{\phi}_{1}-\lambda_{1} \check{s}_{1}\right)+\sigma_{\phi_{1}}^{2} \bar{p}_{1}\right] /\left[\sigma_{\phi_{1}}^{2}+\sigma_{p_{1}}^{2}\right]$

It is a weighted average of the time-averaged SD data, adjusted for the known ambiguities. The $m$-vector $\check{s}_{1}$ contains the SD ambiguities that correspond with the integer $\mathrm{DD}$ ambiguities in $\check{a}_{1}$. Thus $\check{a}_{1}=D^{T} \check{s}_{1}$. The variance matrix of Eq. (7) of course only describes the precision of $\breve{b}$ when the ambiguities indeed may be 
assumed known and non-stochastic. A comparison of the two baseline variance matrices in Eqs. (6) and (7) shows that the baseline variance has been improved by a factor $\epsilon /(1+\epsilon)$. Thus the gain in baseline precision will be very large when $\epsilon$ is small. This in fact, summarizes in a nutshell the whole purpose of ambiguity fixing for short observation time-spans.

Before turning to the least-squares ambiguities, we first digress briefly to consider three orthogonal projectors. They will play an important role in our further analysis.

2.1.4 Three orthogonal projectors Consider the following:

$$
\begin{aligned}
& P=D\left(D^{T} D\right)^{-1} D^{T} \\
& Q=P \bar{A}\left(\bar{A}^{T} P \bar{A}\right)^{-1} \bar{A}^{T} P \\
& R=\left(\bar{A}, e_{m}\right)\left[\left(\bar{A}, e_{m}\right)^{T}\left(\bar{A}, e_{m}\right)\right]^{-1}\left(\bar{A}, e_{m}\right)^{T}
\end{aligned}
$$

It is easily verified that these three matrices are indeed projectors. We already met the first projector earlier. It projects onto the range space $R(D)$ and along $R(D)^{\perp}$. The projector $Q$ projects onto $R(P \bar{A})$ and along $R(P \bar{A})^{\perp}$, and the projector $R$ projects onto $R\left(\bar{A}, e_{m}\right)$ and along $R\left(\bar{A}, e_{m}\right)^{\perp}$. Since $R\left(\bar{A}, e_{m}\right)=R\left(P \bar{A}, e_{m}\right)$ and $R(P \bar{A}) \perp$ $R\left(e_{m}\right)=R(D)^{\perp}$, it follows that $R^{m}$ can be written as the direct sum

$R^{m}=R(D)^{\perp} \oplus R(P \bar{A}) \oplus R\left(\bar{A}, e_{m}\right)^{\perp}$

All these three subspaces are mutually orthogonal and their dimensions are, respectively, equal to 1,3 and $(m-4)$. The projector $I_{m}-P$ is associated with the first subspace, the projector $Q$ with the second and the projector $I_{m}-R$ with the third subspace. Hence, the three orthogonal projectors $P^{\perp}=I_{m}-P, \quad Q$ and $R^{\perp}=I_{m}-R$ sum up to the identity matrix

$I_{m}=P^{\perp}+Q+R^{\perp}$

Some other properties of the three projectors that will be used frequently, are

$$
\begin{aligned}
& P Q=Q P=Q \\
& P R=R P=Q \\
& Q^{\perp}=P^{\perp}+R^{\perp} \\
& P Q^{\perp}=P R^{\perp}
\end{aligned}
$$

Let us now consider the least-squares ambiguities. Using the floated-baseline solution, the least-squares ambiguities follow as

$\hat{a}_{1}=\frac{1}{\lambda_{1}} D^{T}\left[\bar{\phi}_{1}-\bar{A} \hat{b}\right]$

Compare this solution with the ambiguity solution Eq. (2) of the geometry-free model. Since $D^{T} Q D=$ $D^{T} \bar{A}\left(\bar{A}^{T} P \bar{A}\right)^{-1} \bar{A}^{T} D$, an application of the error propagation law to Eq. (12) gives
$Q_{\hat{a}_{1}}=\frac{\sigma_{\phi}^{2}}{\lambda_{1}^{2} k} D^{T}\left[I_{m}+\frac{1}{\epsilon} Q\right] D$

Compare this result with Eq. (3). Instead of the unit matrix in Eq. (3), we now have the projector $Q$ in Eq. (13). This shows that $Q_{\hat{a}_{1}} \leq Q_{\tilde{a}_{1}}$ and thus that the precision of the ambiguities of the time-averaged model is indeed better than the precision of the ambiguities of the geometry-free model. An alternative way of comparing the two variance matrices is to rewrite Eq. (13) as

$Q_{\hat{a}_{1}}=\frac{\sigma_{\phi}^{2}}{\lambda_{1}^{2} k} D^{T}\left[\left(1+\frac{1}{\epsilon}\right) I_{m}-\frac{1}{\epsilon} R^{\perp}\right] D$

This follows from substitution of $Q=I_{m}-P^{\perp}-R^{\perp}$ into Eq. (13), recognizing that $P^{\perp} D=0$. This result shows that the difference between $Q_{\tilde{a}_{1}}$ and $Q_{\hat{a}_{1}}$ is governed by $R^{\perp} / \epsilon$. Since $R^{\perp}=0$ when $m=4$, the two variance matrices are identical in case satellite redundancy is absent. In case satellite redundancy is present however, then $R^{\perp} \neq 0$ and $R^{\perp} / \epsilon$ will have large entries due to the poor precision of the code observables. This shows that the precision of the ambiguities of the time-averaged model will generally be far better than the precision of their counterparts of the geometry-free model.

\subsection{The dual-frequency case}

We will now assume that the GPS observables are available on both frequencies $L_{1}$ and $L_{2}$. The precision of the $L_{2}$ observables is assumed to be identical to the precision of their $L_{1}$ counterparts.

2.2.1 Geometry-free model In the dual-frequency case, the single-baseline model that dispenses with the receiver-satellite geometry reads

$$
\begin{aligned}
& D^{T} \phi_{1}(i)=D^{T} r(i)+\lambda_{1} a_{1} \\
& D^{T} \phi_{2}(i)=D^{T} r(i)+\lambda_{2} a_{2} \\
& D^{T} p_{1}(i)=D^{T} r(i) \\
& D^{T} p_{2}(i)=D^{T} r(i)
\end{aligned}
$$

The redundancy has now increased from $(m-1)(k-1)$ to $(m-1)(3 k-2)$. Thus we now also have redundancy for each single epoch. This stems from the presence of the code data on the second frequency. The leastsquares estimates of the ambiguities can be obtained as follows. Per epoch, one first takes the average of $p_{1}(i)$ and $p_{2}(i)$. This will give the epoch estimate of the range $r(i)$. The epoch estimates of the ambiguities are then obtained from subtracting the range estimate from the phase vectors $\phi_{1}(i)$ and $\phi_{2}(i)$. The least-squares estimates of the ambiguities follow then from a simple averaging over time as

$$
\begin{aligned}
& \tilde{a}_{1}=\frac{1}{\lambda_{1}} D^{T}\left[\bar{\phi}_{1}-\bar{p}\right] \\
& \tilde{a}_{2}=\frac{1}{\lambda_{2}} D^{T}\left[\bar{\phi}_{2}-\bar{p}\right]
\end{aligned}
$$


with $\bar{p}$ the average of $\bar{p}_{1}$ and $\bar{p}_{2}$. An application of the error propagation law gives the ambiguity variancecovariance matrices as

$Q_{\tilde{a}_{1}}=\frac{\sigma_{\phi}^{2}}{\lambda_{1}^{2} k}\left(1+\frac{1}{2 \epsilon}\right) D^{T} D$

$Q_{\tilde{a}_{1} \tilde{a}_{2}}=\frac{\sigma_{\phi}^{2}}{\lambda_{1} \lambda_{2} k} \frac{1}{2 \epsilon} D^{T} D$

$Q_{\tilde{a}_{2}}=\frac{\sigma_{\phi}^{2}}{\lambda_{2}^{2} k}\left(1+\frac{1}{2 \epsilon}\right) D^{T} D$

Compare this result with the single-frequency result of Eq. 3. The precision of the ambiguities is still dominated by the poor precision of the code data. But due to the presence of the dual-frequency data, the variance has improved by a factor of approximately one-half.

2.2.2 Time-averaged model The dual-frequency timeaveraged model reads

$D^{T} \bar{\phi}_{1}=D^{T} \bar{A} b+\lambda_{1} a_{1}$

$D^{T} \bar{\phi}_{2}=D^{T} \bar{A} b+\lambda_{2} a_{2}$

$D^{T} \bar{p}_{1}=D^{T} \bar{A} b$

$D^{T} \bar{p}_{2}=D^{T} \bar{A} b$

The redundancy has increased by $(m-1)$, from $(m-4)$ to $(2 m-5)$. One word of caution however. One should not conclude from this that redundancy exists only when $m \geq 3$. It already exists for $m \geq 2$, due to the presence of the dual-frequency code observables. Thus the redundancy equals $(m-1)$ when $m \leq 4$ and it equals $(2 m-5)$ when $m \geq 4$. But $m \geq 4$ must of course still hold, in order to be able to solve for the model. The matrix $D^{T} \bar{A}$ will be rank defect when $m=3$.

The least-squares solutions for the floated and fixed baseline, together with their variance matrices, follow as

$\hat{b}=\left(\bar{A}^{T} P \bar{A}\right)^{-1} \bar{A}^{T} P \bar{p} ; \quad \check{b}=\left(\bar{A}^{T} P \bar{A}\right)^{-1} \bar{A}^{T} P \bar{q}$

$Q_{\hat{b}}=\frac{\sigma_{p}^{2}}{2 k}\left(\bar{A}^{T} P \bar{A}\right)^{-1} ; \quad Q_{\breve{b}}=\frac{\sigma_{p}^{2}}{2 k} \frac{\epsilon}{1+\epsilon}\left(\bar{A}^{T} P \bar{A}\right)^{-1}$

where $\bar{q}$ is the average of $\bar{q}_{1}$ and $\bar{q}_{2}$. Thus the baseline variance has been improved by a factor of one-half with respect to the single-frequency case, but the ratio between the variance of the fixed and floated baseline has remained equal to $\epsilon /(1+\epsilon)$.

The least-squares ambiguities follow as

$$
\begin{aligned}
& \hat{a}_{1}=\frac{1}{\lambda_{1}} D^{T}\left[\bar{\phi}_{1}-\bar{A} \hat{b}\right] \\
& \hat{a}_{2}=\frac{1}{\lambda_{2}} D^{T}\left[\bar{\phi}_{2}-\bar{A} \hat{b}\right]
\end{aligned}
$$

and their variance-covariance matrices as

$$
\begin{aligned}
& Q_{\hat{a}_{1}}=\frac{\sigma_{\phi}^{2}}{\lambda_{1}^{2} k} D^{T}\left[I_{m}+\frac{1}{2 \epsilon} Q\right] D \\
& Q_{\hat{a}_{1} \hat{a}_{2}}=\frac{\sigma_{\phi}^{2}}{\lambda_{1} \lambda_{2} k} \frac{1}{2 \epsilon} D^{T} Q D \\
& Q_{\hat{a}_{2}}=\frac{\sigma_{\phi}^{2}}{\lambda_{2}^{2} k} D^{T}\left[I_{m}+\frac{1}{2 \epsilon} Q\right] D
\end{aligned}
$$

This result can be compared with Eq. (16) in a similar way as the single-frequency result Eq. (13) was compared with Eq. (3). Now that we have the ambiguity variance matrices available, we are in a position to analyse the characteristics of the ambiguity search space.

\section{The ambiguity search space}

In this section we will study the ambiguity search space, thereby making use of the ambiguity variance matrices of the previous section. First we will study the volume of the ambiguity search space. This is done Sect. 3.1 both for the geometry-free model as well as for the timeaveraged model. Then we will study the use of the widelane ambiguity. In Sect. 3.2 we will show under what conditions the precision of the widelane ambiguities is superior to that of the ordinary DD ambiguities. Finally in Sect. 3.3, we will derive the eigenvalue spectrum of the ambiguity search space. The signature of the spectrum provides a basis for understanding the characteristics of the search for the integer least-squares ambiguities.

\subsection{Volume of search space}

The single-frequency ambiguity search space of the timeaveraged model is defined as

$\left(\hat{a}_{1}-a_{1}\right)^{T} Q_{\hat{a}_{1}}^{-1}\left(\hat{a}_{1}-a_{1}\right) \leq \chi^{2}$

It is a scaled version of the confidence ellipsoid of $\hat{a}_{1}$. The search space is centred at $\hat{a}_{1}$, its shape is governed by the ambiguity variance matrix $Q_{\hat{a}_{1}}$ and its size can be controlled through $\chi^{2}$. The dual-frequency version of the ambiguity search space follows from replacing $a_{1}$ and $\hat{a}_{1}$ by, respectively, $a=\left(a_{1}^{T}, a_{2}^{T}\right)^{T}$ and $\hat{a}$. Their counterparts for the geometry-free model follow from replacing the hat ". " by the tilde " ?".

The integer least-squares ambiguity vector $\check{a}_{1}$ is defined as the minimizer over the set of integers $Z^{m-1}$ of the quadratic form on the left-hand side of Eq. (21). The search space plays a central role in the search for $\check{a}_{1}$. In the literature, many important contributions have been made in the area of GPS integer ambiguity estimation. Examples of proposed methods, together with suggested refinements, can be found in Hatch $(1982,1991)$, Blewitt (1989), Euler and Goad (1990), Frei and Beutler (1990), Frei (1991), Wübbena (1991), Euler and Landau (1992), Abidin (1993), Dedes and Goad (1994), Euler and Hatch (1994).

In Teunissen (1993), the least-squares ambiguity decorrelation adjustment (LAMBDA) was introduced as a 
method for efficiently solving the integer ambiguity estimation problem. The approach taken with this method is to reparametrize the integer least-squares problem such that an equivalent problem is obtained, but one that is much easier to solve. The method consists of two steps. First, an ambiguity transformation is constructed that tries to decorrelate the ambiguities. This decorrelating ambiguity transformation is constructed on the basis of integer approximations to conditional leastsquares transformations. The actual computation of the integer least-squares estimates, but now of the transformed ambiguities, is done in the second step of the method. It is based on a sequential conditional leastsquares adjustment of the ambiguities, that allows one to describe the ambiguity search space as a sum of independent squares. The search is based on this sum of squares and its efficiency stems from the low correlation and high precision of the transformed ambiguities. Studies of the methods performance can be found in Teunissen (1994), Teunissen et al. (1994), Tiberius and de Jonge (1995) and Teunissen et al. (1997).

In Teunissen (1993), the volume of the ambiguity search space was proposed as indicator for the number of grid points inside the search space, thus allowing one to set a suitable value for $\chi^{2}$ and to infer a priori whether one is likely to have a successful validation of the integer least-squares ambiguities or not. For more details on these two issues, including the algorithmic aspects, we refer to Teunissen et al. (1997) and de Jonge and Tiberius (1996).

The volume of the ambiguity search space is proportional to the square root of the determinant of the ambiguity variance matrix. Furthermore, since the decorrelating ambiguity transformation of the LAMBDA method is volume preserving, the volume of the original ambiguity search space is identical to the volume of the transformed search space. Hence, in order to determine the volume of the transformed search space, we do not need the transformed ambiguity variance matrix. Instead, we can restrict our attention to the variance matrices of the original ambiguities. Also note that the determinant is invariant to any admissible ambiguity transformation possible (Teunissen, 1995b). It is independent, for instance, of the choice of reference satellite used in defining the DD ambiguities. This implies that the determinant of the ambiguity variance matrix, as opposed to its trace, truly measures the intrinsic characteristics of the precision of the ambiguities. In the following we will derive easy-to-evaluate, closed-form expressions for the determinants of the four variance matrices $Q_{\tilde{a}_{1}}, Q_{\tilde{a}}, Q_{\hat{a}_{1}}$ and $Q_{\hat{a}}$. We start with the geometry-free model.

3.1.1 Geometry-free model First we derive the determinant for the single-frequency case. In order to derive the determinant $\left|Q_{\tilde{a}_{1}}\right|$ of Eq. (3), we need to obtain the determinant of $D^{T} D$. The diagonal entries of this matrix are all equal to 2 and all its off-diagonal entries are equal to 1 . Hence, $D^{T} D=I_{m-1}+e_{m-1} e_{m-1}^{T}$. With this expression it is easily verified that $D^{T} D$ has $(m-2)$ eigenvalues which are all equal to 1 , and only one eigenvalue which is equal to $m$. Thus we have $\left|D^{T} D\right|=m$. With this result, the determinant of Eq. (3) follows as

$\left|Q_{\tilde{a}_{1}}\right|=m\left(\frac{\sigma_{\phi}^{2}}{\lambda_{1}^{2} k}\right)^{m-1}\left(1+\frac{1}{\epsilon}\right)^{m-1}$

with $m \geq 2$. Note that $m$ can be taken equal to 2 in this formula, while more satellites are available and tracked. In that case the integer ambiguity estimation is tackled on the basis of individual DD ambiguities. We also recognize $m(.)^{m-1}$ as a derivative, which is due to the differencing of the SD observables.

The preceding determinant equals the product of a very small and a very large term. The small term is dominated by the phase variance, whereas the large term is dominated by the ratio of the code variance with the phase variance. As a result the product will be dominated by the variance of code. This shows that the determinant will be very large indeed, unless a sufficient number of epochs are taken into account.

In order to derive the determinant for the dual-frequency case, we first note that $\left|Q_{\tilde{a}}\right|=\left|Q_{\tilde{a}_{1}}\right|\left|Q_{\tilde{a}_{2} \mid \tilde{a}_{1}}\right|$, where the last matrix is the variance matrix of the $L_{2}$ ambiguities conditioned on the $L_{1}$ ambiguities. Both determinants, $\left|Q_{\tilde{a}_{1}}\right|$ and $\left|Q_{\tilde{a}_{2} \mid \tilde{a}_{1}}\right|$, can be derived as Eq. (22) was derived. As a result we get

$\left|Q_{\tilde{a}}\right|=m^{2}\left(\frac{\sigma_{\phi}^{2}}{\lambda_{1}^{2} k}\right)^{m-1}\left(\frac{\sigma_{\phi}^{2}}{\lambda_{2}^{2} k}\right)^{m-1}\left(1+\frac{1}{\epsilon}\right)^{m-1}$

with $m \geq 2$. This result, when compared to Eq. (22), now clearly shows the very beneficial role played by the inclusion of the second frequency. The very large term $(1+1 / \epsilon)^{m-1}$ of Eq. (22) is not squared, but instead is simply copied in Eq. (23). However, apart from the change in wavelength, the very small term is squared in Eq. (23). Hence, one can expect the dual-frequency determinant, when taken to the appropriate power, to be much smaller than its single-frequency counterpart. The phenomenon which we see here at work can be explained by the extreme correlation which exists between the $L_{1}$ and $L_{2}$ ambiguities. Refer to Eq. (16).

3.1.2 Time-averaged model We will now derive the determinants of $Q_{\hat{a}_{1}}$ and $Q_{\hat{a}}$ for the time-averaged model. It follows from Eq. (13) that

$$
\begin{aligned}
\left|Q_{\hat{a}_{1}}\right| & =\left(\frac{\sigma_{\phi}^{2}}{\lambda_{1} k}\right)^{m-1}\left|D^{T}\left[I_{m}+\frac{1}{\epsilon} Q\right] D\right| \\
& =m\left(\frac{\sigma_{\phi}^{2}}{\lambda_{1} k}\right)^{m-1}\left|I_{m-1}+\frac{1}{\epsilon} D^{+} Q D\right| \\
& =m\left(\frac{\sigma_{\phi}^{2}}{\lambda_{1} k}\right)^{m-1}\left|I_{m}+\frac{1}{\epsilon} Q P\right|
\end{aligned}
$$

where $D^{+}=\left(D^{T} D\right)^{-1} D^{T}$ is the pseudo-inverse of $D$. To obtain the last equality in this triple, use has been made 
of the fact that $|I+M N|=|I+N M|$ for any two matrices $M$ and $N$ of appropriate order. Since $Q P=Q$ and $Q$ is a projector of rank 3 , it follows that $\left|I_{m}+Q P / \epsilon\right|=(1+1 / \epsilon)^{3}$ and therefore that

$\left|Q_{\hat{a}_{1}}\right|=m\left(\frac{\sigma_{\phi}^{2}}{\lambda_{1} k}\right)^{m-1}\left(1+\frac{1}{\epsilon}\right)^{3}$

with $m \geq 4$. This is a remarkable result. It shows that the determinant of $Q_{\hat{a}_{1}}$ and thus also the volume of the ambiguity search space Eq. (21), is independent of the relative receiver-satellite geometry. The same holds true in the dual-frequency case. Since the last two determinants in $\left|Q_{\hat{a}}\right|=\left|Q_{\hat{a}_{1}}\right|\left|Q_{\hat{a}_{2} \mid \hat{a}_{1}}\right|$ can be derived in a similar way as Eq. (24) has been derived, we obtain for the dual-frequency case

$\left|Q_{\hat{a}}\right|=m^{2}\left(\frac{\sigma_{\phi}^{2}}{\lambda_{1} k}\right)^{m-1}\left(\frac{\sigma_{\phi}^{2}}{\lambda_{2} k}\right)^{m-1}\left(1+\frac{1}{\epsilon}\right)^{3}$

with $m \geq 4$. When compared to Eq. (24), we also notice again the beneficial role played by the inclusion of the second frequency.

3.1.3 Single-epoch geometry-based model Since the timeaveraged model reduces to the single-epoch geometrybased model for $k=1$, the determinant of the ambiguity variance matrix of the latter model is also independent of the receiver-satellite geometry. Since the geometry does not need to be known, the volume is extremely simple to evaluate. Hence, for applications where use is made of instantaneous ambiguity resolution techniques, the preceding formulae also provide a very simple way of estimating the number of grid points inside the ambiguity search space. A numerical study that shows how well the volume of the ambiguity search space approximates the number of grid points contained in it, is presented in Teunissen et al. (1996).

Although the single-frequency and dual-frequency determinants here do not depend on the receiver-satellite geometry, they still feature an important property which is shared by the time-averaged model and the geometrybased model, but which is not shared with the geometryfree model. This is the time-invariance of the baseline versus the change in time of the receiver-satellite ranges. Due to the time-invariance of the baseline, the large term $(1+1 / \epsilon)$ is only taken to the third power, whereas in Eqs. (22) and (23), it is taken to the power of $(m-1)$. This shows that the two determinants $\left|Q_{\tilde{a}_{1}}\right|$ and $\left|Q_{\hat{a}_{1}}\right|$, or the two determinants $\left|Q_{\tilde{a}}\right|$ and $\left|Q_{\hat{a}}\right|$, are equal when satellite redundancy is absent, but that those of the geometry-free model are significantly larger when more than four satellites are tracked. This thus clearly signifies the beneficial role played by satellite redundancy in case of the geometry-based model and the time-averaged model.

\subsection{On the widelane ambiguity}

Here we will study, both for the geometry-free model as well as for the time-averaged model, the precision of the celebrated widelane ambiguity. This will be done relative to the precision of the DD ambiguities. The leastsquares estimate of the widelane ambiguity vector will be denoted as $\hat{a}_{w}$. In order to compare the precision of the widelane ambiguities with the precision of the $L_{1}$ ambiguities, we compare the variances of the two linear functions $f^{T} \hat{a}_{w}$ and $f^{T} \hat{a}_{1}$. The variance of $f^{T} \hat{a}_{w}$ is smaller than, or at the most equal to, the variance of $f^{T} \hat{a}_{1}$, for any arbitrary function $f \in R^{m-1}$, if and only if the maximum eigenvalue of the generalized eigenvalue problem

$\left|Q_{\hat{a}_{w}}-\mu Q_{\hat{a}_{1}}\right|=0$

is less than or equal to 1 . To solve this eigenvalue problem, we need to know the variance matrices $Q_{\hat{a}_{w}}$ and $Q_{\hat{a}_{1}}$. Substitution of $I_{m}=P^{\perp}+Q+R^{\perp}$ into $Q_{\hat{a}_{1}}$ of Eq. (20) gives

$Q_{\hat{a}_{1}}=\frac{\sigma_{\phi}^{2}}{\lambda_{1}^{2} k} D^{T}\left[\left(1+\frac{1}{2 \epsilon}\right) Q+R^{\perp}\right] D$

The least-squares estimate of the widelane ambiguity vector is defined as $\hat{a}_{w}=\hat{a}_{1}-\hat{a}_{2}$. An application of the error propagation law gives $Q_{\hat{a}_{w}}=Q_{\hat{a}_{1}}+Q_{\hat{a}_{2}}-2 Q_{\hat{a}_{1} \hat{a}_{2}}$, since the covariance matrix of the $L_{1}$ and $L_{2}$ ambiguities is symmetric as well. Substitution of the variancecovariance matrices of Eq. (20) and again using $I_{m}=P^{\perp}+Q+R^{\perp}$, gives

$Q_{\hat{a}_{w}}=\frac{\sigma_{\phi}^{2}}{\lambda_{1}^{2} k} D^{T}\left[r Q+s R^{\perp}\right] D$

with

$r=s+\frac{1}{2 \epsilon}\left(1-\frac{\lambda_{1}}{\lambda_{2}}\right)^{2}$ and $s=1+\frac{\lambda_{1}^{2}}{\lambda_{2}^{2}}$

Note that these two variance matrices reduce to their counterparts of the geometry-free model when we set $Q=I_{m}$ and $R^{\perp}=0$.

With the two variance matrices the characteristic equation Eq. (26) can be written as

$\left|D^{T}\left[\left(r-\mu\left(1+\frac{1}{2 \epsilon}\right)\right) Q+(s-\mu) R^{\perp}\right] D\right|=0$

From this it follows, since $Q$ and $R^{\perp}$ are projectors with $R(Q) \perp R\left(R^{\perp}\right)$ and $\operatorname{dim} R(Q)=3, \operatorname{dim} R\left(R^{\perp}\right)=m-4$, that the eigenvalues are given as

$\mu_{1}=\frac{2 \epsilon}{2 \epsilon+1} r \quad(3 \times), \mu_{2}=s \quad((m-4) \times)$

It will be clear that when all these eigenvalues are smaller than 1, all functions of $\hat{a}_{w}$ would have a precision better than that of the same functions of $\hat{a}_{1}$. Hence, in that case the individual widelane ambiguities also would have a 
precision better than their $L_{1}$ counterparts. Conversely, if all eigenvalues are larger than 1, then all functions of $\hat{a}_{w}$ would have a precision which is poorer than the precision of the same functions of $\hat{a}_{1}$.

Let us first consider the $(m-4)$ eigenvalues $\mu_{2}=s$. Since $s>1$, there exist $(m-4)$ linear functions of $\hat{a}_{w}$ that have a precision poorer than the precision of the same functions of $\hat{a}_{1}$. This already shows, contrary to some claims in the literature, that it is not guaranteed that the widelane ambiguities are of a better precision than the $L_{1}$ ambiguities. Fortunately, since the functions that produce $\mu=s$ are of a very high precision when applied to $\hat{a}_{1}$ and since $s$ is only 1.61 , these widelane functions will still be very precise. Since

Let us now consider the remaining three eigenvalues.

$\mu_{1}>1 \Leftrightarrow \epsilon>\left(\frac{\lambda_{2}}{\lambda_{1}}-1\right) \simeq 0.28$

it follows that these remaining eigenvalues are also larger than 1 when $\epsilon$ is large enough. Thus instead of having a better precision, it is then guaranteed that the widelane ambiguities will have a poorer precision than the $L_{1}$ ambiguities. Fortunately, in practice the precision of the code observables is such that the condition $\epsilon<0.28$ is easily met. In fact, $\epsilon$ is so small (e.g. $\epsilon \simeq 10^{-4}$ ), that we may approximate the first three eigenvalues as

$\mu_{1} \simeq\left(1-\frac{\lambda_{1}}{\lambda_{2}}\right)^{2} \simeq 0.05$

This approximation will become poorer though, and the first three eigenvalues will become larger, if the precision of the code observables gets better.

The approximation in Eq. (32) shows that the variance of the widelane functions that correspond with the three eigenvalues $\mu_{1}$, is better by a factor of 20 , than the variance of the corresponding functions of the $L_{1}$ ambiguites. And fortunately, this improvement is applied to the 'correct' functions, namely those which have a very poor precision in case of the $L_{1}$ ambiguities. The conclusion reads therefore, that by using the widelane ambiguities, functions of the $L_{1}$ ambiguities that have a very poor precision have their variance improved by a factor of about 20, while functions of the $L_{1}$ ambiguities that already have a very high precision, have their variance degraded by a factor of only 1.61 .

The preceding analysis was based on the time-averaged model. For the geometry-free model we have to set $Q=I_{m}$ and $R^{\perp}=0$ in Eq. (29). Hence, in this case all $(m-1)$ eigenvalues are equal to $\mu_{1}$. This shows that in contrast to the time-averaged model, all functions of the widelane ambiguities now have a better precision than the same functions of $\hat{a}_{1}$, provided of course that $\epsilon$ is sufficiently small. The conclusion reads therefore for the geometry-free model, that it is guaranteed that the widelane ambiguities are of a better precision than the $L_{1}$ ambiguities. For the time-averaged model, it is not guaranteed. The difference in these two results is due to the receiver-satellite geometry.
This improvement in precision, when going from the original DD ambiguities to the widelane ambiguities, does not explain in itself why it makes sense to use the widelane ambiguities instead of the ordinary DD ambiguities. In this context it is of particular importance to know what happens to the correlation between the widelane ambiguities and the remaining ambiguities. Thus in order to understand the relevance of the widelane ambiguities properly, one should consider all the $2(m-1)$ ambiguities together, and not only the subset of $(m-1)$ widelane ambiguities. In order to study the characteristics of the set of $2(m-1)$ ambiguities, we will analyse the eigenvalue spectra of the ambiguity variancecovariance matrices. This is done in the next section.

\subsection{The eigenvalue spectra}

The dual-frequency ambiguity search space can be represented as

$$
\begin{aligned}
\left(\hat{a}_{1}-a_{1}\right)^{T} & Q_{\hat{a}_{1}}^{-1}\left(\hat{a}_{1}-a_{1}\right) \\
& +\left(\hat{a}_{2 \mid 1}-a_{2}\right)^{T} Q_{\hat{a}_{2} \mid \hat{a}_{1}}^{-1}\left(\hat{a}_{2 \mid 1}-a_{2}\right) \leq \chi^{2}
\end{aligned}
$$

where $\hat{a}_{2 \mid 1}$ is the least-squares estimate of $a_{2}$ conditioned on $a_{1}$. The eigenvalue analysis of the variance-covariance matrices to be presented in this subsection, allows us, apart from visualizing the geometry of the quadratic forms involved in Eq. (33), to explain the behaviour of search halting, which is typically experienced when solving for the $L_{1}$ and $L_{2}$ ambiguities. Furthermore, it will allow us to show how the efficiency in the search can be improved by transforming to new and less correlated ambiguities. The use of the three projectors $P^{\perp}, Q$ and $R^{\perp}$ greatly facilitates the eigenvalue analysis, since they in fact already provide the appropriate subspace decomposition.

3.3.1 The single-frequency case We will first consider the single-frequency case of the time-averaged model. Thus the second quadratic form of Eq. (33) will be absent for the moment. Substitution of Eq. (10) into Eq. (13) gives

$Q_{\hat{a}_{1}}=\frac{\sigma_{\phi}^{2}}{\lambda_{1}^{2} k} D^{T}\left[P^{\perp}+\left(1+\frac{1}{\epsilon}\right) Q+R^{\perp}\right] D$

Since the range spaces of the three projectors $P^{\perp}, Q$ and $R^{\perp}$ decompose $R^{m}$ into orthogonal subspaces, and since the matrix $D Q_{\hat{a}_{1}}^{-1} D^{T}$ equals the pseudo-inverse of the variance matrix of the SD $L_{1}$ ambiguities, the orthonormal columns of these three projectors determine the principal axes of the ambiguity search space in $R^{m}$. Hence, the squared lengths of the $(m-1)$ principal axes of the ambiguity search space in the subspace $R(D)$ follow directly from Eq. (34) as

$$
\underbrace{\frac{\sigma_{\phi}^{2}}{\lambda_{1}^{2} k}\left(1+\frac{1}{\epsilon}\right)}_{3 \times}, \underbrace{\frac{\sigma_{\phi}^{2}}{\lambda_{1}^{2} k}}_{(m-4) \times}
$$


This result shows that we have a very large discontinuity in the eigenvalue spectrum. Since $\epsilon$ will be very small, the first three eigenvalues are very much larger than the remaining $(m-4)$ eigenvalues. Hence, the ambiguity search space will be extremely elongated. It is due to this large elongation and the fact that the principal axes are not aligned with the grid axes, that one will have difficulties in efficiently solving for the integer leastsquares estimates of the DD ambiguities (Teunissen 1993, 1995a).

In order to compare the preceding spectrum with the single-frequency spectrum of the geometry-free model, we set $Q=I_{m}$ and $R^{\perp}=0$ in Eq. (34). From this, the eigenvalue spectrum immediately follows as

$$
\underbrace{\frac{\sigma_{\phi}^{2}}{\lambda_{1}^{2} k}\left(1+\frac{1}{\epsilon}\right)}_{3 \times}, \underbrace{\frac{\sigma_{\phi}^{2}}{\lambda_{1}^{2} k}\left(1+\frac{1}{\epsilon}\right)}_{(m-4) \times}
$$

Hence, all eigenvalues are equal, and there is no discontinuity in the spectrum. This shows that one will not have any difficulties in computing the integer leastsquares estimates of the DD ambiguities for the geometry-free model. This however does not imply that the spectrum Eq. (36) should be preferred over Eq. (35). Here it is important to make a clear distinction between the integer estimation problem and the integer validation problem. Furthermore, it is important to make a clear distinction between whether one solves for the DD ambiguities or for transformed ambiguities, as it is done in the LAMBDA method. A smooth spectrum without a discontinuity is beneficial for the numerical solution of the integer estimation problem. But a discontinuity in the spectrum, such as in the spectrum of Eq. (35) with its $(m-4)$ very small eigenvalues, is beneficial for the integer validation problem. This explains why validation is so much easier with the time-averaged model and the geometry-based model than with the geometry-free model. It is due to these small eigenvalues that the determinant of the ambiguity variance matrix and thus the volume of the ambiguity search space is pulled down to smaller values. The small eigenvalues in the spectrum can also be used to pull down the large values in the spectrum, provided one transforms to new and decorrelated ambiguities. We will make this clear in a short while, by example of the widelane ambiguities. First however, we will consider the dual-frequency spectrum of the DD ambiguities.

3.3.2 The dual-frequency case In the dual-frequency case we have, in analogy with Eq. (34), the ambiguity variance matrices

$$
\begin{aligned}
& Q_{\hat{a}_{1}}=\frac{\sigma_{\phi}^{2}}{\lambda_{1}^{2} k} D^{T}\left[P^{\perp}+\left(1+\frac{1}{2 \epsilon}\right) Q+R^{\perp}\right] D \\
& Q_{\hat{a}_{2} \mid \hat{a}_{1}}=\frac{\sigma_{\phi}^{2}}{\lambda_{2}^{2} k} D^{T}\left[P^{\perp}+\left(1+\frac{1}{2 \epsilon+1}\right) Q+R^{\perp}\right] D
\end{aligned}
$$

Hence, in this case the spectrum of the time-averaged model reads

$$
\underbrace{\frac{\sigma_{\phi}^{2}}{\lambda_{1}^{2} k}\left(1+\frac{1}{2 \epsilon}\right)}_{3 \times}, \underbrace{\frac{\sigma_{\phi}^{2}}{\lambda_{1}^{2} k}}_{(m-4) \times}, \underbrace{\frac{\sigma_{\phi}^{2}}{\lambda_{2}^{2} k}\left(1+\frac{1}{2 \epsilon+1}\right)}_{3 \times}, \underbrace{\frac{\sigma_{\phi}^{2}}{\lambda_{2}^{2} k}}_{(m-4) \times}
$$

When we set $Q=I_{m}$ and $R^{\perp}=0$, the corresponding spectrum for the geometry-free model follows as

$$
\begin{aligned}
\underbrace{\frac{\sigma_{\phi}^{2}}{\lambda_{1}^{2} k}\left(1+\frac{1}{2 \epsilon}\right)}_{3 \times}, \underbrace{\frac{\sigma_{\phi}^{2}}{\lambda_{1}^{2} k}\left(1+\frac{1}{2 \epsilon}\right)}_{(m-4) \times}, \underbrace{\frac{\sigma_{\phi}^{2}}{\lambda_{2}^{2} k}\left(1+\frac{1}{2 \epsilon+1}\right)}_{3 \times}, & \underbrace{\frac{\sigma_{\phi}^{2}}{\lambda_{2}^{2} k}\left(1+\frac{1}{2 \epsilon+1}\right)}_{(m-4) \times}
\end{aligned}
$$

Compare Eqs. (38) and (39) with Eqs. (35) and (36), respectively. Again we note a large discontinuity in the spectrum of the time-averaged model. The first three eigenvalues are large, while the remaining $(2 m-5)$ are small. But now we also notice a discontinuity in the spectrum of the geometry-free model. The first $(m-1)$ eigenvalues are all equal and large, while the remaining $(m-1)$ eigenvalues are all equal but very small. Thus we see here the impact at work of having data on a second frequency available.

3.3.3 Ambiguity transformations In order to show how the discontinuity in the spectrum can be used to our advantage, the transformation to the widelane ambiguities will serve as an example. The ambiguity transformation matrix that transforms the DD ambiguities into the widelane and $L_{2}$ ambiguities, reads

$Z_{1}=\left[\begin{array}{cc}I_{m-1} & -I_{m-1} \\ 0 & I_{m-1}\end{array}\right]$

This transformation is volume preserving and thus leaves the product of the eigenvalues invariant. The distribution of the eigenvalues within the spectrum will change however. We will first consider the spectrum of the geometry-free model. By setting $Q=I_{m}$ and $R^{\perp}=0$ in

$$
\begin{aligned}
& Q_{\hat{a}_{w}}=\frac{\sigma_{\phi}^{2}}{\lambda_{1}^{2} k} D^{T}\left[P^{\perp}+r Q+s R^{\perp}\right] D \\
& Q_{\hat{a}_{2} \mid \hat{a}_{w}}=\frac{\sigma_{\phi}^{2}}{\lambda_{2}^{2} k} D^{T}\left[P^{\perp}+\left(1+\frac{1}{\epsilon}\right) \frac{1}{r} Q+\frac{1}{s} R^{\perp}\right] D
\end{aligned}
$$

the eigenvalues immediately follow as

$$
\underbrace{\frac{\sigma_{\phi}^{2}}{\lambda_{1}^{2} k} r}_{3 \times}, \underbrace{\frac{\sigma_{\phi}^{2}}{\lambda_{1}^{2} k} r}_{(m-4) \times}, \underbrace{\frac{\sigma_{\phi}^{2}}{\lambda_{2}^{2} k}\left(1+\frac{1}{\epsilon}\right) \frac{1}{r}}_{3 \times}, \underbrace{\frac{\sigma_{\phi}^{2}}{\lambda_{2}^{2} k}\left(1+\frac{1}{\epsilon}\right) \frac{1}{r}}_{(m-4) \times}
$$


Compare this result with Eq. (39). It shows that the ambiguity transformation has reduced the gap between the large and small eigenvalues. With $\epsilon$ small, the gap has been changed from approximately

$\frac{\sigma_{\phi}^{2}}{\lambda_{1}^{2} k} \frac{1}{2 \epsilon} \quad$ to $\quad \frac{\sigma_{\phi}^{2}}{\lambda_{1}^{2} k} \frac{0.05}{2 \epsilon}$

A similar effect is achieved for the time-averaged model. The spectrum of eigenvalues follows from Eq. (40) as

$\underbrace{\frac{\sigma_{\phi}^{2}}{\lambda_{1}^{2} k} r}_{3 \times}, \underbrace{\frac{\sigma_{\phi}^{2}}{\lambda_{1}^{2} k} s}_{(m-4) \times}, \underbrace{\frac{\sigma_{\phi}^{2}}{\lambda_{2}^{2} k}\left(1+\frac{1}{\epsilon}\right) \frac{1}{r}}_{3 \times}, \underbrace{\frac{\sigma_{\phi}^{2}}{\lambda_{2}^{2} k} \frac{1}{s}}_{(m-4) \times}$

This shows, when compared to Eq. (38), that a similar reduction in the gap between the large and small eigenvalues is achieved, while at the same time, the differences between the other eigenvalues have remained small.

The preceding phenomenon is due to the fact that the widelane ambiguities are less correlated with the $L_{2}$ ambiguities than the $L_{1}$ ambiguities are, provided $\epsilon$ is small enough. This can be explained by means of the following two inequalities

$Q_{\hat{a}_{2} \mid \hat{a}_{w}}>Q_{\hat{a}_{2} \mid \hat{a}_{1}}, Q_{\hat{a}_{1}}>Q_{\hat{a}_{w}}$

The first inequality follows from $Q_{\hat{a}_{2} \hat{a}_{1}} Q_{\hat{a}_{1}}^{-1} Q_{\hat{a}_{1} \hat{a}_{2}}$ $>Q_{\hat{a}_{2} \hat{a}_{w}} Q_{\hat{a}_{w}}^{-1} Q_{\hat{a}_{w} \hat{a}_{2}}$, which expresses the fact that the widelane ambiguities are less correlated with the $L_{2}$ ambiguities than the $L_{1}$ ambiguities are. The second inequality follows from the first and the fact that ambiguity transformations need to be volume preserving. The two inequalities show that the last $(m-1)$ very small eigenvalues are pulled up, thus allowing the first $(m-1)$ eigenvalues, including the very large ones, to be pulled down. As a result, the spectrum becomes flatter with a smaller gap between the very large and very small eigenvalues.

The transformation to the widelane ambiguities is one of the many choices one has for using ambiguities other than the original DD ambiguities (Teunissen 1995b). However, it is not an optimal choice. It is not difficult to show that the following two ambiguity transformations

$Z_{2}=\left[\begin{array}{ll}4 & -5 \\ 1 & -1\end{array}\right] \otimes I_{m-1}, Z_{3}=\left[\begin{array}{cc}-60 & 77 \\ -7 & 9\end{array}\right] \otimes I_{m-1}$

already do a better job than $Z_{1}$ in flattening the spectrum, thereby bringing the large eigenvalues down to smaller values. Examples hereof are given in Teunissen et al. (1994).

But even though these two ambiguity transformations do a better job, they are generally not optimal either. These types of ambiguity transformation have two restrictions. First, they can only be applied when dual-frequency data are available. Secondly, they fail to take care of the existing receiver-satellite geometry. Both these restrictions are absent in the LAMBDA method. In fact, it is the additional information on the receiver- satellite geometry which is used with this method so as to bring the spectrum down to even smaller values.

\section{Summary}

In this contribution we studied the time-averaged model in its relation with the geometry-free model and the geometry-based model. In particular, the ambiguity precision, the volumes of the ambiguity search spaces and the eigenvalue spectra of the ambiguity variance covariance matrices were analysed.

The models considered are of the single-baseline type, separated by a short distance only. Hence, it was assumed that the DD GPS observables are sufficiently insensitive to orbital uncertainties in the fixed orbits and to residual ionospheric and tropospheric delays. We also assumed that time correlation is absent and that no correlation exists between the phase and code observables.

The precision of the ambiguities of the time-averaged model 'interpolates' between the precision of the ambiguities of the geometry-free model and that of the geometry-based model. In the absence of satellite redundancy, the precision equals that of the geometryfree model, and in the absence of multi-observation epochs, the precision equals that of the geometry-based model. However, in the presence of satellite redundancy and multi-observation epochs, the ambiguity precision of the time-averaged model is better than that of the geometry-free model, but poorer than that of the geometry-based model. Although the least-squares estimators of the time-averaged model are generally less optimal than those of the geometry-based model, they can be considered to be close to optimal in case of short observation time-spans.

We studied the precision of the widelane ambiguities in relation to the precision of the $L_{1} \mathrm{DD}$ ambiguities. The conditions were identified under which the precision of the widelane ambiguities is guaranteed to be better than the precision of the $L_{1}$ ambiguities. For most practical purposes the conclusion reads for the singleepoch geometry-based and the time-averaged model, that by using the widelane ambiguities, functions of the $L_{1}$ ambiguities that have a poor precision have their variance improved by a factor of about 20 , while functions of the $L_{1}$ ambiguities that already have a very high precision, have their variance degraded by a factor of only 1.61. This conclusion depends however on the assumption that the precision of the code observables is far poorer than the precision of the phase observables.

Table 1. The determinants of the ambiguity variance matrices $\left(\epsilon=\sigma_{\phi}^{2} / \sigma_{p}^{2}\right)$.

\begin{tabular}{lll}
\hline$\left|Q_{a}\right|$ & geometry-free $(m \geq 2)$ & time-averaged $(m \geq 4)$ \\
\hline$L_{1}$ & $m\left(\frac{\sigma_{\phi}^{2}}{\lambda_{1}^{2} k}\right)^{m-1}\left(1+\frac{1}{\epsilon}\right)^{m-1}$ & $m\left(\frac{\sigma_{\phi}^{2}}{\lambda_{1}^{2} k}\right)^{m-1}\left(1+\frac{1}{\epsilon}\right)^{3}$ \\
$L_{1} / L_{2}$ & {$\left[m\left(\frac{\sigma_{\phi}^{2}}{\lambda_{1} \lambda_{2} k}\right)^{m-1}\right]^{2}\left(1+\frac{1}{\epsilon}\right)^{m-1}$} & {$\left[m\left(\frac{\sigma_{\phi}^{2}}{\lambda_{1} \lambda_{2} k}\right)^{m-1}\right]^{2}\left(1+\frac{1}{\epsilon}\right)^{3}$} \\
\hline
\end{tabular}


Table 2. Eigenvalue spectra as defined in Sect. 3.3 for the geometry-free and the time-averaged models

\begin{tabular}{lllll}
\hline $\begin{array}{l}\text { eigen- } \\
\text { values }\end{array}$ & $\begin{array}{l}\sigma_{\phi}^{2} \\
\lambda_{1}^{2} k\end{array}()$. & $(m-4) \times$ & $\frac{\sigma_{\phi}^{2}}{\lambda_{2}^{2} k}()$. & \\
& $3 \times$ & $\left(1+\frac{1}{\epsilon}\right)$ & $3 \times$ & - \\
\hline geometry-free & $\left(1+\frac{1}{\epsilon}\right)$ & $\left(1+\frac{1}{2 \epsilon}\right)$ & - & $\left(1+\frac{1}{2 \epsilon+1}\right)$ \\
$L_{1}$ & $\left(1+\frac{1}{2 \epsilon}\right)$ & $\left(1+\frac{1}{2 \epsilon}\right) \mu_{1}$ & $\left(1+\frac{1}{2 \epsilon+1}\right)$ & $\left(1+\frac{1}{2 \epsilon+1}\right) / \mu_{1}$ \\
$L_{1} / L_{2}$ & $\left(1+\frac{1}{2 \epsilon}\right) \mu_{1}$ & 1 & & - \\
$L_{w} / L_{2}$ & $\left(1+\frac{1}{\epsilon}\right)$ & 1 & - & 1 \\
time-averaged & $\left(1+\frac{1}{2 \epsilon}\right)$ & $\mu_{2}$ & $\left(1+\frac{1}{2 \epsilon+1}\right)$ & $1 / \mu_{1}$ \\
$L_{1}$ & $\left(1+\frac{1}{2 \epsilon}\right) \mu_{1}$ & $\left(1+\frac{1}{2 \epsilon+1}\right) / \mu_{1}$ & \\
$L_{1} / L_{2}$ & & &
\end{tabular}

The volume of the ambiguity search space is an indicator for the number of grid points inside the search space. The volume is proportional to the square root of the determinant of the ambiguity variance matrix. Easyto-evaluate, closed-form expressions were derived for the determinants of the ambiguity variance matrices. This was done for the single-frequency case and for the dual-frequency case of the time-averaged model and the geometry-free model. For easy reference, these results are summarized in Table 1. The determinant of the variance matrix is known as a generalized variance (Stuart and Ord 1991) and when taken to the appropriate power, $1 /(m-1)$ for the single-frequency case and $1 / 2(m-1)$ for the dual-frequency case, it also equals the geometric mean of the eigenvalues. The formulae of the table clearly show the beneficial role played by satellite redundancy and dual-frequency data. The table shows for instance, when recognizing that $\sigma_{\phi} \ll \sigma_{p}$ and when using the appropriate power, that the singlefrequency case of the geometry-free model differs to a first order by a factor of $\frac{\lambda_{1}}{\lambda_{2}} \frac{\sigma_{\phi}}{\sigma_{p}}$ from the dual-frequency case of the same model. This signifies the impact of using a second frequency. Similarly, we have a factor of $\left(\frac{\sigma_{\phi}}{\sigma_{p}}\right)^{\frac{m-4}{m-1}}$ when going from the dual-frequency geometryfree model to the dual-frequency time-averaged model. This signifies the impact of satellite redundancy.

With the results of Table 1 , the volumes of the ambiguity search spaces can be computed as $V=$ $\chi^{n} U_{n} \sqrt{\left|Q_{a}\right|}$, where $Q_{a}$ is the ambiguity variance matrix, $n$ its order, and $U_{n}$ the volume of the unit-sphere in $R^{n}$. The volume of the unit-sphere is given as $U_{n}=\pi^{n / 2} / \Gamma(n / 2+1)$, where $\Gamma(x)$ is the gamma-function, which can be evaluated by means of the recurrence relation $\Gamma(x+1)=x \Gamma(x)$, for $x>0$, and the initial values $\Gamma(1 / 2)=\sqrt{\pi}$ and $\Gamma(1)=1$.

We also derived and analysed the lengths of the principal axes of the respective ambiguity search spaces. The eigenvalue analysis allowed us to explain the phenomenon of search halting, which is typically experienced when solving for the $L_{1}$ and $L_{2}$ ambiguities. We also discussed how the efficiency of the search can be improved by transforming to new and less correlated ambiguities. This was shown by means of the widelane ambiguities as an example. For easy reference the sets of eigenvalues are summarized in Table 2.
Acknowledgements. This work was supported by the Alexander von Humboldt Stiftung and finalized during the author's sabbatical stay at the University of Stuttgart (Germany) with Professor E. Grafarend as his host.

\section{References}

Abidin HA (1993) Computational and geometric aspects of on-thefy ambiguity resolution. Dept Surv Eng Tech Rep 164, University of New Brunswick, Fredericton, New Brunswick, Canada

Blewitt G (1989) Carrier-phase ambiguity resolution for the Global Positioning System applied to geodetic baselines up to $2000 \mathrm{~km}$. J Geophys Res 94: 12187-12203

Dedes G, Goad C (1994) Real-time cm-level GPS positioning of cutting blade and earth movement equipment. In: Proc 1994 Nat Tech Meeting ION, San Diego, California. pp 587-594

Euler H-J, Goad C (1990) On optimal filtering of GPS dual-frequency observations without using orbit information. Bull Geod 65: $130-143$

Euler H-J, Hatch R (1994) Comparison of several AROF kinematic techniques. Proc ION-94: 363-370

Euler H-J, Landau H (1992) Fast GPS ambiguity resolution onthe-fly for real-time applications. In: Proc 6th Int Geod Symp Satellite Positioning. Columbus, Ohio, 17-20 March 1992. pp 650-729

Frei E (1991) Rapid differential positioning with the global positioning system. Schweizerischen Geodatischen Kommission, Band 44, Bern, Switzerland

Frei E. Beutler G (1990) Rapid static positioning based on the Fast Ambiguity Resolution Approach FARA: theory and first results. Manuscr Geod, 15:325-356

Hatch R (1982) The synergism of GPS code and carrier measurements. In: Proc 3rd Int Geod Symp Satellite Positioning. Las Vegas, New Mexico, 8-12 February, 1982, Vol. 2. pp 1213-1231

Hatch R (1991) Instantaneous ambiguity resolution. In: Kinematic systems in geodesy, surveying and remote sensing, IAG Symposia 127. Springer, Berlin Heidelberg New York pp 299-308

de Jonge PJ, Tiberius CCJM (1996) The LAMBDA method for integer ambiguity estimation: implementation aspects. $L G R-S e r$ 12, Delft Geodetic Computing Centre, Delft

Stuart A. Ord JK (1991) Advanced theory of statistics, Vol. 2. Hodder and Stoughton, London.

Teunissen PJG (1993) Least-squares estimation of the integer GPS ambiguities. LGR-Ser 6, Delft Geodetic Computing Centre, Delft

Teunissen PJG (1994) A new method for fast carrier phase ambiguity estimation. In: Proc IEEE PLANS'94, Las Vegas, 11-15 April. pp 562-573

Teunissen PJG (1995a) The least-squares ambiguity decorrelation adjustment: a method for fast GPS integer ambiguity estimation. $J$ Geod 70: 65-82 
Teunissen PJG (1995b) The invertible GPS ambiguity transformations. Manuscr Geod 20: 489-497

Teunissen PJG (1996) An analytical study of ambiguity decorrelation using dual-frequency code and carrier phase. J Geod, 70:515-528

Teunissen PJG (1997) The geometry-free GPS ambiguity search space in weighted ionosphere. $J$ Geod: in print

Teunissen PJG, de Jonge PJ, Tiberius CCJM (1994) On the spectrum of the GPS DD ambiguities. In: Proc ION GPS-94, Salt Lake City, UT, 20-23 September. pp 115-124

Teunissen PJG, de Jonge PJ, Tiberius CCJM (1997) The leastsquares ambiguity decorrelation adjustment: its performance on short baselines. $J$ Geod. in print
Teunissen PJG, de Jonge PJ, Tiberius CCJM (1996) The volume of the GPS ambiguity search space and its relevance for integer ambiguity resolution. In: ION GPS-96, Kansas City, Missouri, 17-20 September. pp 889-898

Tiberius CCJM, de Jonge PJ (1995) Fast positioning using the LAMBDA method. In: Proc DSNS95, Bergen, Norway 24-28 April. Paper 30, 8 pp

Wübbena G (1991) Zur Modellerung von GPS Beobachtungen für die Hochgenaue Positionsbestimmung. Universität Hannover, Germany 